\title{
Study of the X-ray radiation interaction with a multislit collimator for the creation of microbeams in radiation therapy
}

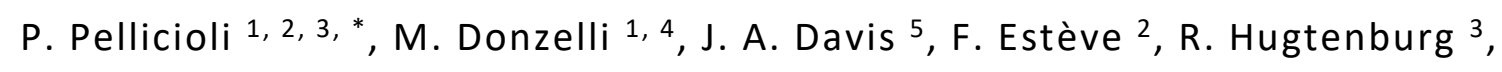
S. Guatelli ${ }^{5}$, M. Petasecca ${ }^{5}$, M. L. F. Lerch ${ }^{5}$, E. Bräuer-Krisch ${ }^{1}$, M. Krisch 1, 2.

1. ESRF - The European Synchrotron Radiation facility, ID17 Biomedical beamline, Grenoble, France.

2. STROBE - Synchrotron Radiation for Biomedicine, Grenoble, France.

3. Swansea University Medical School, Singleton Park, Swansea, United Kingdom.

4. ICR - The Institute of Cancer Research, London, United Kingdom.

5. University of Wollongong, School of Physics, Wollongong, Australia.

* Corresponding author: Paolo Pellicioli, paolo.pellicioli@esrf.fr

Paper accepted and published on:

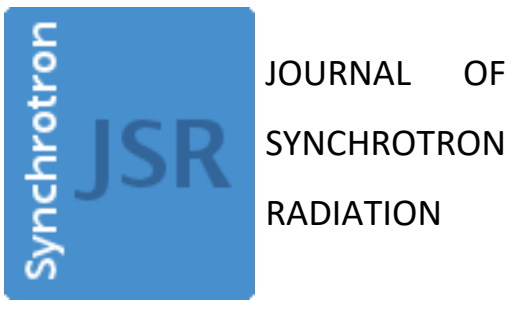

ISSN: $1600-5775$

Volume 28| Part 2| March 2021| Pages 392-403

https://doi.org/10.1107/S1600577520016811 
Microbeam radiation therapy (MRT) is a developing radiotherapy, based on the use of beams only few tens of micrometer wide, generated by synchrotron X-ray sources. The spatial fractionation of the homogenous beam into an array of microbeams is possible using a multislit collimator (MSC), i.e. a machined metal block with regular apertures. Dosimetry in MRT is challenging and previous works still 25 show differences between calculated and experimental dose profiles of $10 \%-30 \%$, which are not acceptable for a clinical implementation of the treatment. The interaction of the X-rays with the MSC may contribute to the observed discrepancies; the present study therefore investigates the dose contribution due to radiation interaction with the MSC inner walls and radiation leakage of the MSC.

Dose distributions inside a water-equivalent phantom were evaluated for different field sizes and three typical spectra used for MRT studies at the European Synchrotron Biomedical beamline ID17. Film dosimetry was utilized to determine the contribution of radiation interaction with the MSC inner walls; Monte Carlo simulations were implemented to calculate the radiation leakage contribution.

Both factors turned out to be relevant for the dose deposition, especially for small fields. Photons interacting with the MSC walls may bring up to $16 \%$ more dose in the valley regions, between the 35 microbeams. Depending on the chosen spectrum, the radiation leakage close to the phantom surface can contribute up to $50 \%$ of the valley dose for a $5 \times 5 \mathrm{~mm}^{2}$ field.

Our study underlines that a detailed characterization of the MSC must systematically be performed and accurate MRT dosimetry protocols must include the contribution of radiation leakage and radiation interaction with the MSC in order to avoid significant errors in the dose evaluation at the micrometric scale. 


\section{INTRODUCTION}

The idea to spatially fractionate the radiation field into a grid of smaller beams of about $1 \mathrm{~mm}$ size to reduce side effects in the patients' skin (Laissue et al., 2012) was introduced soon after the beginning of X-ray radiation therapy (1957). The advent of synchrotron X-ray sources and technological progress made possible the creation of microbeams, only few tens of micrometers wide, which proved to be extremely effective in radiotherapy. The fundamental biological effect at the basis of microbeam radiation therapy (MRT) is the dose-volume effect, i.e. the capability of healthy tissues to tolerate a high dose of radiation if it is confined into small volumes (Zeman et al., 1961).

In the past decades, a large body of pre-clinical work has shown the potential and enhanced effectiveness of MRT compared to other conventional techniques based on homogeneous radiation fields (Laissue et al., 1998, 2013; Schültke et al., 2008; Slatkin et al., 2007; Van Der Sanden et al., 2010; Smyth et al., 2018; Bouchet et al., 2013; Crosbie et al., 2010; Bouchet et al., 2010; Ibahim et al., 2016, 2014; Slatkin et al., 1992; Slatkin, Spanne et al., 1995; Fernandez-Palomo et al., 2020). While initially MRT research focused on applications for cancer treatment, recent studies extended to the treatment of chronic pain and epilepsies (Zippo et al., 2019; Romanelli \& Bravin, 2011; Studer et al., 2015; Pouyatos et al., 2016; Anschel et al., 2011; Pouyatos et al., 2013).

MRT studies were performed with arrays of $25-100 \mu \mathrm{m}$ wide beams, spaced by $100-1000 \mu \mathrm{m}$ (centerto-center) to understand differences in tissues response (Bräuer-Krisch et al., 2010; Dilmanian et al., 2004; Serduc et al., 2009; Griffin et al., 2012; Laissue et al., 1998). The most commonly used geometry is an array of $50 \mu \mathrm{m}$ beams with a $400 \mu \mathrm{m}$ pitch. This array is divided into peak regions corresponding to the microbeams, and valley regions where the dose arises only from scattered photons inside the target. The ratio between peak and valley doses is called peak-to-valley dose ratio (PVDR). Typically, the valley dose is kept below the limit established for conventional radiotherapy, while the peak dose can be as high as several hundreds of grays, with dose rates up to $16 \mathrm{kGy} / \mathrm{s}$. A steep dose gradient at the edge of peak regions is achievable using orthovoltage X-ray, with a mean energy in the order of $100 \mathrm{keV}$. Due to the small vertical divergence of synchrotron X-rays, radiation fields of sufficient vertical size are obtained scanning the target vertically through the beam, while the horizontal fan of a wiggler source allows for horizontal field sizes of $40-100 \mathrm{~mm}$.

To transform a homogeneous radiation field into a spatially fractionated array of microbeams, a multislit collimator (MSC) is placed in front of the target. The first MSC at fixed geometry was realized alternating 
Au and Al foils (Slatkin, Dilmanian et al., 1995; Archer, 1998; Bräuer-Krisch et al., 2003) but it was soon replaced by a more versatile Tecomet ${ }^{\circledR}$ model able to define microbeams of variable width (Bräuer-Krisch et al., 2005). Improved machining techniques allowed the realization of more precise and accurate apertures and two of the most recent MSCs are described in the work of E. Bräuer-Krisch et al. (BräuerKrisch et al., 2009). While variable aperture MSCs are more versatile, an MSC with fixed geometry is easier to align and to control during an experiment. Nowadays, MSCs are typically made of a tungsten-based alloy, machined out of a single piece or assembled using individual blades to obtain the desired width and spacing of the microbeam array.

At present, there are several dedicated beamlines for MRT research in operation world-wide, at the ESRF (Grenoble, France), the Australian Synchrotron (Clayton, Australia), SPring-8 (Hyogo, Japan), and the Canadian Light Source (Saskatoon, Canada). In parallel, compact radiation sources with adequate characteristics are under development (Bartzsch et al., 2016; Hadsell et al., 2014; Bartzsch et al., 2020). The Australian Synchrotron and the ESRF move forward towards phase-I clinical trials and therefore undertake significant efforts to develop reliable dosimetry protocols capable to predict the absorbed dose in the target. Differences between calculated and measured doses in MRT are often between 10 and 30\%, still significantly higher than the differences considered acceptable in conventional radiotherapy. One possible source of this discrepancy is the interaction of X-rays with the MSC which has never been investigated in detail. The slightly divergent photon beam interacts with the inner surfaces of the MSC, 90 giving rise to scattering effects. Furthermore, the metal blades may be not able to completely stop the incident radiation and a significant amount of photons might be transmitted.

So far, only a partial study of these two aspects have been conducted. A Monte Carlo (MC) model of the MSC was presented by Nettelbeck et al., which included the influence of the beam divergence on the penumbra at the edge of the microbeams (Nettelbeck et al., 2009) but an experimental evaluation was not performed. Moreover, the study of possible radiation leakage was omitted due to, at the time, long simulations required to decrease the uncertainty in the valley dose. Martínez-Rovira et al. developed a MC model of the ID17 beamline using Shadow and Penelope codes (Martínez-Rovira et al., 2012). The simulated PVDR were often higher than experimental values, with differences above $10 \%$. Bartzsch et al. performed MC simulations with the Geant4 toolkit including synchrotron beam polarization and suggested an approximate model for beam divergence and MSC leakage (Bartzsch et al., 2014). The influence of the photon interactions with the MSC inner walls was observed by Fournier et al. using a silicon strip detector (Fournier, Cornelius et al., 2016). Changing the aperture alignment by $\pm 0.02^{\circ}$, the 
detector revealed valley dose variations, but a quantitative dosimetry study was not performed. More recently, Ocadiz et al. have found dose differences above 25\% (Ocadiz et al., 2019) when comparing film dosimetry with a MC hybrid dose calculation (Donzelli et al., 2018) in MRT.

For the Imaging and Medical Beamline (IMBL) of the Australian Synchrotron, two MC models have been recently presented. A. Dipuglia et al. (Dipuglia et al., 2019) developed a detailed MC model of the entire $I M B L$, including the electron transport inside the wiggler source, for the generation of phase space files as input for dose calculation. L. Day et al. (Day et al., 2020) presented as well a reliable IMBL model, where an analytical approach is used to calculate the generated polychromatic spectrum that is used in MC simulations for the dose calculation. Both works reported convincing experimental data in support to their models. The approach used in this study is closer to the one proposed by L. Day et al., as the spectrum profile is generated by an analytical approach using XOP software (see section 2.1.1). The MSC available at the IMBL has similar properties to the MSC available at the ID17 beamline because both were realized using the same construction technique. The ID17 X-ray spectrum has an overall higher photon flux, a higher mean energy, and extends to higher photon energies than the IMBL spectrum. The detailed comparison between the spectra used at the ID17 beamline and the IMBL is not part of the manuscript, but a different contribution of radiation leakage can be expected between the two beamlines.

The purpose of this work is to investigate the discrepancies between MC simulations and experimental 120 dosimetry related to the X-ray interaction with the MSC. Radiochromic film dosimetry was used to quantify the parasitic radiation component due to the photons interaction with the inner walls of the MSC while MC simulations based on Geant4 (Agostinelli et al., 2003) were used to quantify the influence of the MSC radiation leakage into the valley region.

\section{MATERIALS AND METHODS}

\subsection{Experimental setup at the ESRF ID17 Biomedical beamline}

\subsubsection{Spectra definition}

The synchrotron radiation source at the ESRF ID17 Biomedical beamline is a wiggler of $1.5 \mathrm{~m}$ length and a magnetic field of $1.6 \mathrm{~T}$ at a gap of $24.8 \mathrm{~mm}$. This is the typical configuration used for MRT experiments. The X-ray beam is transported mostly in vacuo up to the target that is placed around $40.5 \mathrm{~m}$ from the source point. A detailed description of the ID17 beamline can be found in several publications (Cornelius et al., 2014; Crosbie et al., 2015; Martínez-Rovira et al., 2012). Here, only the relevant information for the current study will be presented. 
The X-ray spectrum generated by the wiggler was calculated with the XOP software version 2.3 (Del Río \& Dejus, 2004). The spectrum at the target is modified due to the attenuation of several components in the 135 beam path, for which the Beer-Lambert law was applied, and material attenuation coefficients from the NIST database (Hubbell \& Seltzer, 1996) were taken.

For this study, three different attenuation configurations were utilized. Configuration 1, or conventional MRT configuration, is the historically and originally used configuration for MRT experiments at ID17. Configuration 2, or pre-clinical MRT configuration, has been introduced to satisfy the request of having

140 less dose deposition in dense tissues, such as bones, during veterinary pre-clinical studies. Compared to the conventional MRT configuration, the pre-clinical one presents a harder spectrum. The spectrum hardening is obtained by using different material and material thickness combination of five attenuators. This extra filtration produces an intrinsic reduction of the spectrum intensity as well. Configuration 3, or clinical MRT configuration, answers to more rigid safety rules that must be considered in a realistic clinical 145 scenario where extra components such as krypton filters (Requardt et al., 2013) and ionization chambers must be added to monitor the beam during patient irradiation. While the energy characteristics of the clinical spectrum are close to the pre-clinical one, the clinical spectrum is further reduced in intensity due to the additional components added along the beam path.

Table 1 reports the list of all the absorbers used to define the beam quality of the three MRT irradiation 150 configurations.

Table 2 presents the main characteristics of the three spectra.

Figure 1 presents some properties and relations between the three spectra. Figure $1(A)$ shows the conventional MRT, pre-clinical MRT and clinical MRT X-ray energy spectra profiles impinging on the MSC and Figure 1(B) shows the corresponding profiles obtained after normalization to the maximum intensity, 155 facilitating the recognition of the energy shift. Figure 1 (C) presents the relative intensity difference of the pre-clinical and clinical normalized spectrum with respect to the conventional normalized spectra. The different attenuator combinations reduce the low energy part of the spectrum, below $90 \mathrm{keV}$, but they are not effective for energies above $100 \mathrm{keV}$. The weight contribution of photons with energies above 200 $\mathrm{keV}$ is between 1.6 and 2.4 times more significant for pre-clinical and clinical spectra compared to the 160 conventional spectrum. 
Table 1. List of beam intercepting components and their thickness for the three considered X-ray spectra configurations: conventional MRT, pre-clinical MRT and clinical MRT.

\begin{tabular}{|c|c|c|c|c|c|c|}
\hline \multirow[b]{2}{*}{ Component } & \multicolumn{2}{|c|}{$\begin{array}{c}\text { Configuration } 1 \\
\text { Conventional MRT }\end{array}$} & \multicolumn{2}{|c|}{$\begin{array}{l}\text { Configuration } 2 \\
\text { Pre-clinical MRT }\end{array}$} & \multicolumn{2}{|c|}{$\begin{array}{c}\text { Configuration } 3 \\
\text { Clinical MRT }\end{array}$} \\
\hline & Material & $\begin{array}{l}\text { Thickness } \\
{[\mathrm{mm}]}\end{array}$ & Material & $\begin{array}{l}\text { Thickness } \\
{[\mathrm{mm}]}\end{array}$ & Material & $\begin{array}{l}\text { Thickness } \\
\text { [mm] }\end{array}$ \\
\hline $\begin{array}{l}\text { Vacuum windows } \\
\text { (total) }\end{array}$ & $\mathrm{Be}$ & 2.3 & $\mathrm{Be}$ & 2.3 & $\mathrm{Be}$ & 2.3 \\
\hline Attenuator rack & & & & & & \\
\hline Attenuator 1 & C & 1.15 & C & 1.42 & C & 1.42 \\
\hline Attenuator 2 & Al & 0.28 & $\mathrm{Al}$ & 0.28 & Al & 0.28 \\
\hline Attenuator 3 & Al & 1.24 & Al & 1.24 & $\mathrm{Al}$ & 1.24 \\
\hline Attenuator 4 & $\mathrm{Cu}$ & 0.35 & $\mathrm{Cu}$ & 1.42 & $\mathrm{Cu}$ & 1.42 \\
\hline Attenuator 5 & $\mathrm{Cu}$ & 0.69 & $\mathrm{Cu}$ & 0.69 & $\mathrm{Cu}$ & 0.69 \\
\hline $\begin{array}{l}\text { Krypton filter } \\
\text { Ionization } \\
\text { Chamber (ICO) }\end{array}$ & not used & & not used & & $\begin{array}{c}\mathrm{Kr} \\
\text { (85 mbar) }\end{array}$ & 2197 \\
\hline Windows & not used & & not used & & $\mathrm{Al}$ & $4 \times 0.5$ \\
\hline Window coating & not used & & not used & & $\mathrm{Au}$ & $8 \times \approx 0.0001$ \\
\hline Exit window & Al & 0.5 & $\mathrm{Al}$ & 0.5 & $\mathrm{Al}$ & 0.5 \\
\hline $\begin{array}{l}\text { Ionization } \\
\text { Chamber (ICObis) }\end{array}$ & not used & & not used & & PMMA & 19 \\
\hline
\end{tabular}

165 Table 2. Mean energy and energy of the intensity maximum for the conventional, pre-clinical, and clinical MRT spectra. The last column presents the relative difference of the intensity maximum of the three calculated $X$-ray spectra configurations.

\begin{tabular}{cccc}
\hline $\begin{array}{c}\text { Spectrum } \\
\text { configuration }\end{array}$ & $\begin{array}{c}\text { Mean Energy } \\
{[\mathrm{keV}]}\end{array}$ & $\begin{array}{c}\text { Peak Energy } \\
{[\mathrm{keV}]}\end{array}$ & $\begin{array}{c}\text { Change of the intensity } \\
\text { maximum compared to the } \\
\text { Conventional configuration [\%] }\end{array}$ \\
\hline Conventional & 104.2 & 87.7 & $0 \%$ \\
Pre-clinical & 119.0 & 102.1 & $-48 \%$ \\
Clinical & 122.8 & 108.2 & $-70 \%$ \\
\hline
\end{tabular}




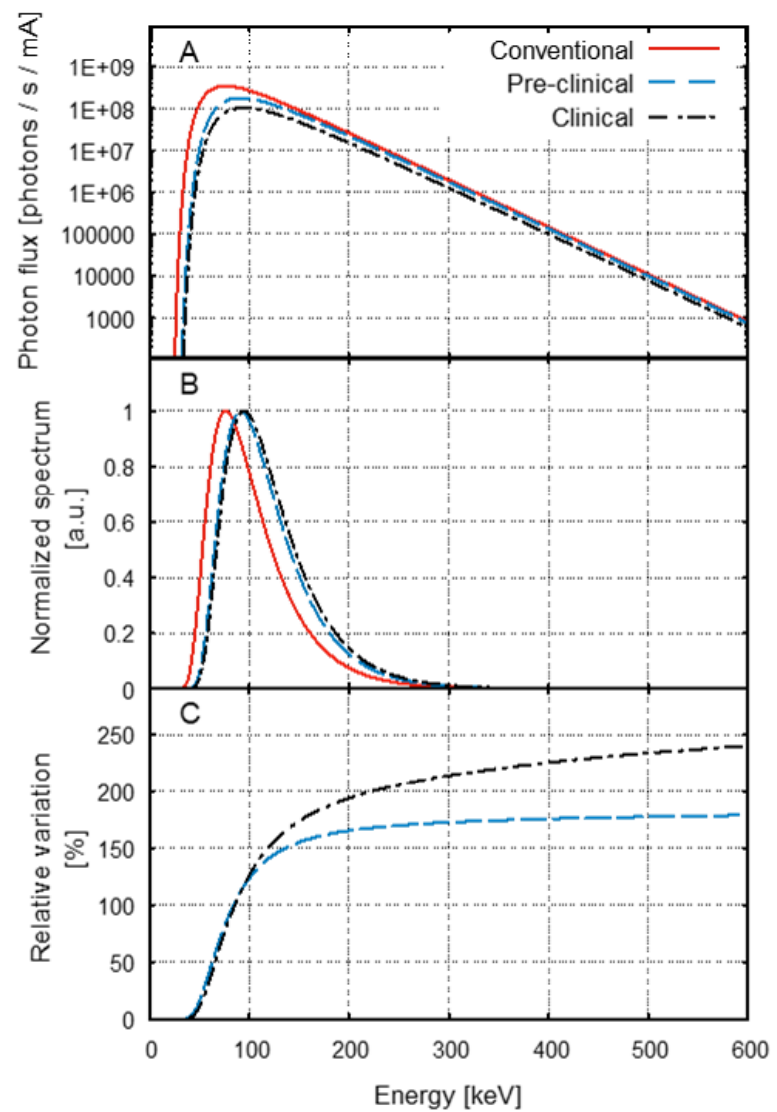

170 Figure 1. (A) Plots of the three X-ray spectra profiles used in the study (conventional MRT, pre-clinical MRT and clinical MRT spectrum) normalized to the machine current and (B) corresponding spectrum profiles after normalization to the respectively maximum intensity value. (C) Plot of the relative differences of the pre-clinical and clinical normalized spectra compared to conventional spectrum.

\subsubsection{Multislit collimator}

175 The multislit collimator transforms the homogeneous field generated by the synchrotron source into a spatially fractionated array of radiation. For this study, the single stack MSC described in the work of E. Bräuer-Krisch et al. (Bräuer-Krisch et al., 2009) was used (see Figure 2). This MSC was made out of 125 single tungsten carbide alloy plates because wire cutting techniques are not precise enough for machining apertures of less than $100 \mu \mathrm{m}$ wide out of a single block. In this way, an array of $50 \mu \mathrm{m}$ wide beams spaced by $400 \mu \mathrm{m}$ was obtained. 

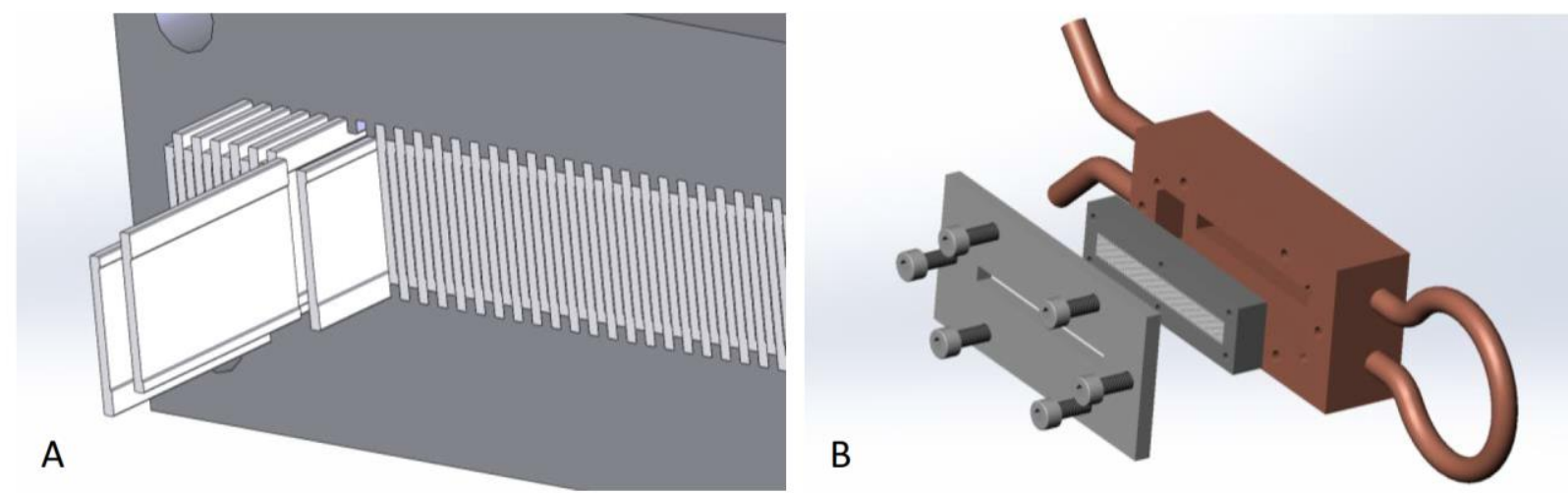

Figure 2. Technical drawings of the single stack MSC used at the ESRF ID17 Biomedical beamline. Panel A shows the configuration used to assemble the single plates inside the metal frame (left); panel (B) reports the geometry of the water-cooling system (right). Courtesy of T. Brochard (ESRF).

The metal base on which the MSC is mounted is fixed on a rotational stage able to align the apertures parallel to the beam with $\pm 0.001^{\circ}$ precision. A water-cooling system and a nitrogen gas flow impinging on the MSC avoid overheating during radiation exposures. The MSC is $8 \mathrm{~mm}$ thick and made of WF20 tungsten-carbide alloy (supplier: JJ x-ray, Denmark) with density of $14.08 \pm 0.01 \mathrm{~g} / \mathrm{cm}^{3}$. The chemical material composition is reported in Table 3.

Table 3. Material composition of the WF20 tungsten-carbide alloy in weight percentage (wt \%). Table reported from E. Bräuer-Krisch et al. (Bräuer-Krisch et al., 2009).

\begin{tabular}{cccc}
\hline & \multicolumn{3}{c}{ WF20 chemical composition } \\
& WC & Co & VC $+\mathrm{Cr}_{3} \mathrm{C}_{2}$ \\
\hline wt \% & $86.4-87.2$ & $11.5-12.5$ & $1.1-1.3$ \\
\hline
\end{tabular}

The total attenuation coefficient of the MSC was calculated in the relevant photon range, $1-600 \mathrm{keV}$, using the NIST database (Hubbell \& Seltzer, 1996). The percentage of transmitted radiation by the MSC was calculated following the Beer-Lambert law and it is reported in Figure 3(A). The metal blades effectively stop all photons with energies below $200 \mathrm{keV}$ but allow the partial transmission of photons with energies above $200 \mathrm{keV}$. For comparison, the percentage of transmitted radiation by a $8 \mathrm{~mm}$ thick pure tungsten carbide (WC) MSC of density $15.63 \mathrm{~g} / \mathrm{cm}^{3}$ and a pure tungsten (W) MSC of density of 19.3 $\mathrm{g} / \mathrm{cm}^{3}$ is as well reported in Figure 3(A).

Figure 3(B) shows the expected spectra arising from the ID17 MSC due to radiation leakage. They were obtained, multiplying the normalized spectra of Figure 1(B) with the WF20 MSC radiation transmission 
profile of Figure $3(A)$. Considering the normalized spectra impinging on the MSC for comparison, the leakage for the clinical spectrum is about a factor 2 higher than for the conventional spectrum.
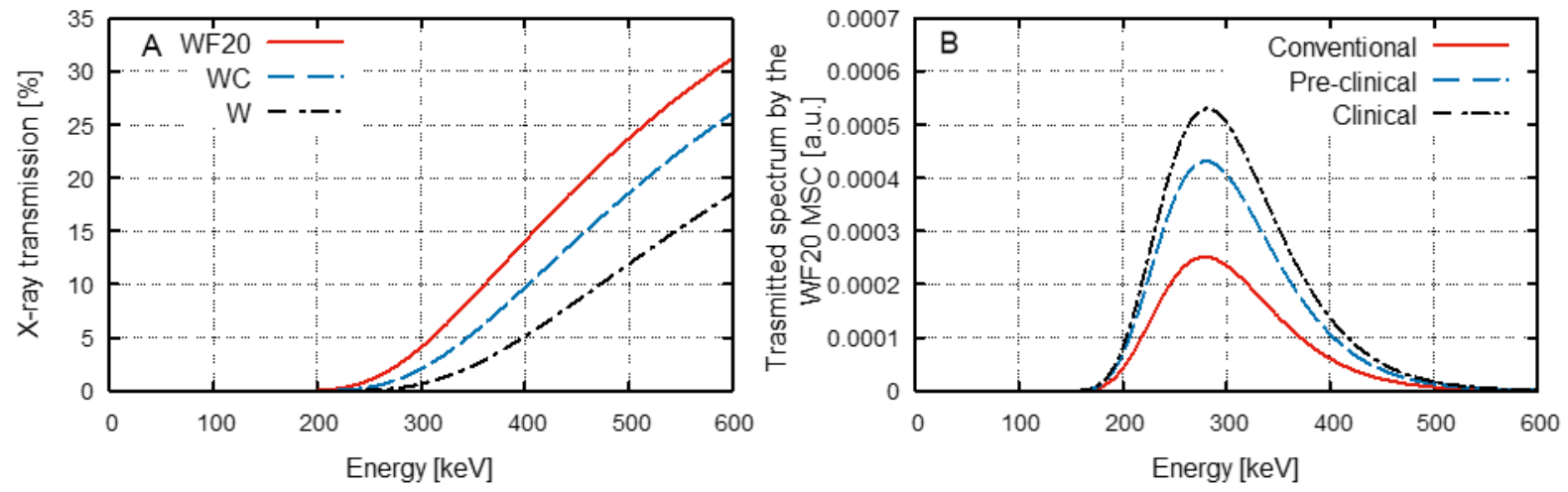

Figure 3. (A) X-ray transmission as a function of photon energy for the ID17 WF20 tungsten carbide alloy MSC compared to a MSC made out of tungsten carbide (WC) or pure tungsten (W). (B) Expected X-ray spectra arising from the incomplete absorption of the ID17 MSC metal blades, obtained multiplying each spectrum profile of Figure 1(B) by the WF 20 MSC transmission curve of Figure 3(A).

\subsection{Film dosimetry for quantification of the radiation interaction with the multislit} collimator inner walls

Radiochromic films are well known detectors in radiation therapy, able to visualize the 2-dimensional dose distribution of the irradiated area. The detailed description of the protocol developed for MRT film dosimetry at micrometric scale and used in this work is documented in the literature (Pellicioli et al., 2019). Here, only the specific irradiation setup and geometry used in the study will be presented.

Prior to film irradiation, absolute dosimetry under reference conditions was performed following the protocol presented by Fournier et al. (Fournier, Crosbie et al., 2016), following as close as possible the recommendations of the International Atomic Energy Agency - IAEA - for reference dosimetry using medium energy kilovoltage X-rays (Musolino \& IAEA TRS-398, 2001). Due to the limited vertical dimension of the synchrotron beam (see Introduction), a PTW PinPoint 31014 ionization chamber was scanned through the beam to evaluate the beam dose rate. Reference dosimetry was performed in a waterequivalent plastic cubic phantom placing the ionization chamber in the center of the scanned $20 \times 20 \mathrm{~mm}^{2}$ broad beam field and with its central axis at $20 \mathrm{~mm}$ depth. Radiochromic films (Gafchromic ${ }^{\mathrm{TM}} \mathrm{HD}-\mathrm{V} 2$ type) were irradiated under the same reference conditions for the definition of the calibration curve between 0 Gy (non-irradiated film) and $250 \mathrm{~Gy}$, the dose-range of interest for the study. 
Following the film calibration, microbeam irradiations were performed placing and aligning the MSC inside the beam. Three radiation fields were defined with a size of $5 \times 5 \mathrm{~mm}^{2}, 10 \times 10 \mathrm{~mm}^{2}$ and $20 \times 20 \mathrm{~mm}^{2}$ as array of $50 \mu \mathrm{m}$ wide beams with a pitch of $400 \mu \mathrm{m}$. A single film irradiation was performed for each field size at $5 \mathrm{~mm}, 10 \mathrm{~mm}, 20 \mathrm{~mm}, 30 \mathrm{~mm}, 50 \mathrm{~mm}$ and $80 \mathrm{~mm}$ depth in the water-equivalent phantom. Films were scanned with an optical microscope 10 days after exposition, following the protocol presented by Pellicioli et al. (Pellicioli et al., 2019). The dose profile of the five central valleys was considered for each microbeam array and dose values were integrated over the central $50 \mu \mathrm{m}$ wide region. The uncertainty related to the relative comparison between valley doses on a single piece of film is due to the microscopic non-homogeneity that a radiochromic film may present over a $2 \times 2 \mathrm{~mm}^{2}$ of area considered in this study. For dose values integrated over the defined valley regions, the uncertainty was found to be $1.52 \%(2 \sigma)$. As the uncertainty value is small, it is not reported in the section 3.1 to facilitate data visualization.

\subsection{Monte Carlo simulations for radiation leakage study}

The investigation of the radiation leakage through the MSC metal blades was performed with MC radiation transport simulations, implemented with Geant4 toolkit version 10.03.p03 using the Penelope physics models (Agostinelli et al., 2003; Salvat et al., 2009). Two scenarios were considered. The first represents the ideal case of a perfect array of parallel microbeams without any radiation contribution due to radiation leakage in the MSC. The second mimics a more realistic irradiation setup, where an ideal broad beam of photons generated at the wiggler source position with the X-ray energy spectrum as described in section 2.1.1 is impinging on the MSC. In this case, the radiation leakage in the MSC is taken into account and all the photons that are not absorbed by the MSC are considered for the calculation of the dose inside the target.

A water equivalent phantom of $100 \times 100 \times 100 \mathrm{~mm}^{3}$ was used as target. Three radiation fields of $5 \times 5$ $\mathrm{mm}^{2}, 10 \times 10 \mathrm{~mm}^{2}$ and $20 \times 20 \mathrm{~mm}^{2}$ were defined as an array of $50 \mu \mathrm{m}$ wide beams of $400 \mu \mathrm{m}$ pitch. The three different X-ray energy spectra (conventional MRT, pre-clinical MRT and clinical MRT) defined in

250 section 2.1.1 were considered. The dose evaluation was performed as an average dose of five central peaks and four central valleys of each array, considering a $10 \mu \mathrm{m}$ central region for each peak and a 50 $\mu \mathrm{m}$ region for each valley. Vertically, along the scanning direction, a $100 \mu \mathrm{m}$ region was considered in the center of the field. The depth dose profile was studied with a spatial resolution of $1 \mathrm{~mm}$ inside the phantom (along the beam propagation direction).

255 To provide a reliable micrometric dose scoring due to the spatial fractionation at micrometric scale of the radiation field, the production threshold for secondary particles was defined as $1 \mu \mathrm{m}$ while the maximum 
step size for electron propagation was set to $5 \mu \mathrm{m}$. The total number of simulated photons was adjusted for each simulation in order to achieve a density of $1.6 \times 10^{6}$ photons $/ \mu \mathrm{m}^{2}$ inside the microbeam region as well as impinging on the MSC in the more realistic scenario. The simulation statistical uncertainties for the total considered regions were found to be less than $0.04 \%(2 \sigma)$ and $0.27 \%(2 \sigma)$ for the peak and valley dose, respectively. As the uncertainty values are very small, they are not reported in the section 3.2 to facilitate data visualization.

To further extend the work, MC simulations were performed to evaluate the effect of different MSC materials in terms of radiation leakage. The case of a pure tungsten carbide (WC) alloy and pure tungsten

265 (W) as defined in section 2.1.2 was chosen, and depth dose profiles were calculated for the three field sizes and spectra mentioned above.

\section{RESULTS}

\subsection{Photon interaction on the inner walls of the multislit collimator}

Figure 4 presents four dose profiles evaluated with the radiochromic films at $5 \mathrm{~mm}, 20 \mathrm{~mm}, 50 \mathrm{~mm}, 80$ $\mathrm{mm}$ depth inside the phantom for the case of a $5 \times 5 \mathrm{~mm}^{2}$ array. It is possible to recognize a pattern of "regular" and "distorted" valleys at all measured depths. This pattern arises from the presence of a radiation contribution in the valley regions due to photons interacting with the inner surfaces of the MSC. Regular valleys have a symmetrical and rounded profile, as expected, while distorted valleys present an additional parasitic radiation contribution on one or both sides, corresponding to an irregular profile characterized by a higher dose. Regular valleys are marked with a blue dot while distorted valleys are marked with a red cross. The same pattern is visible for all the profiles. The same phenomenon is also observed for the $10 \times 10 \mathrm{~mm}^{2}$ and $20 \times 20 \mathrm{~mm}^{2}$ arrays of microbeams. 


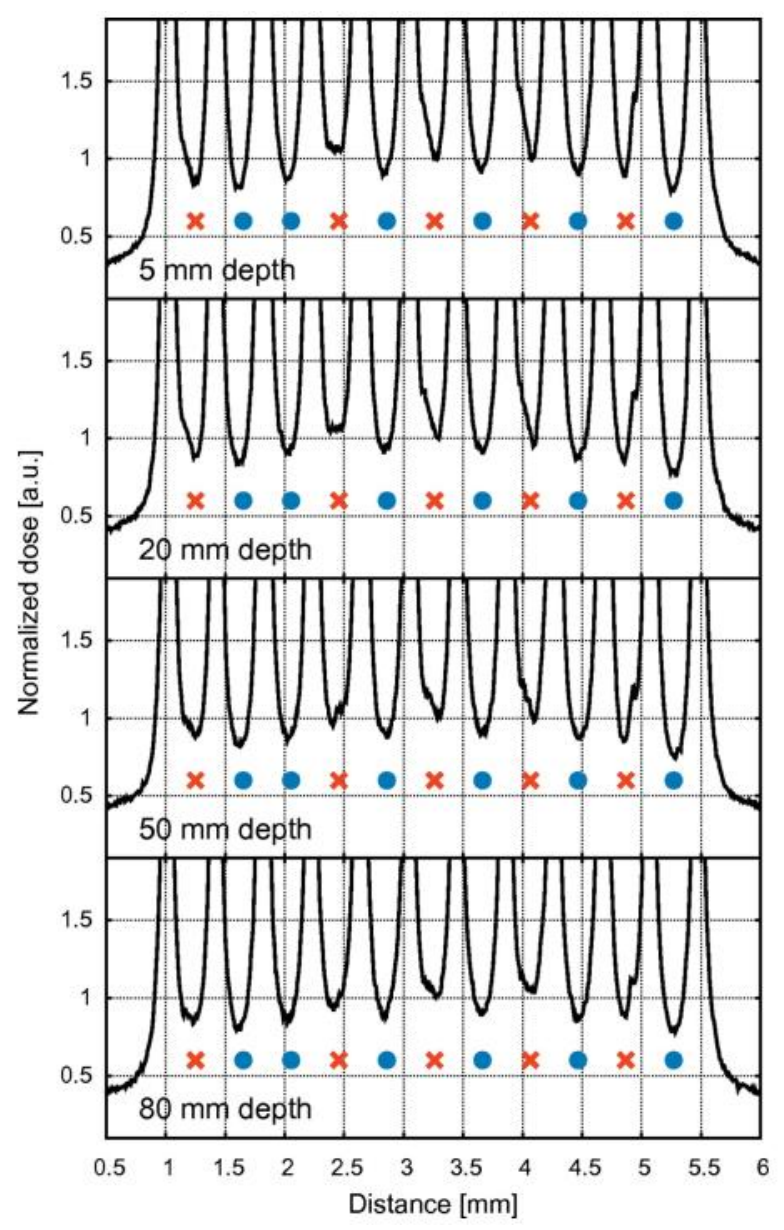

Figure 4. Valley dose profile of a $5 \times 5 \mathrm{~mm}^{2}$ microbeam field at different depths. The repetitive pattern between regular and distorted valley shapes is identified by blue dots and red crosses, respectively. All the dose profiles have been normalized to the minimum dose value of the central valley, in this case a distorted one.

After the visual classification of the valleys, the five central valleys of each irradiated field were considered, and the average dose value was calculated for each regular and distorted valley group. Figure 5 shows the relative dose variation of the distorted valleys with respect to the regular ones and Table 4 reports the relative increase of the dose. The contribution of photons interacting with the MSC walls is more significant for a small field size than for large ones. The amount of parasitic radiation is a constant fraction of the peak dose, while the dose deposited into valley regions due to photon scattering inside the phantom is smaller for small field size. Consequently, the additional dose contribution is more significant in a small field. The proposed linear fit for the three series of data of Figure 5 suggests also that, for large fields, the dose difference between distorted and regular valleys tends to decrease moving deeper inside the phantom, while for the $5 \times 5 \mathrm{~mm}^{2}$ field the difference remains constant. 


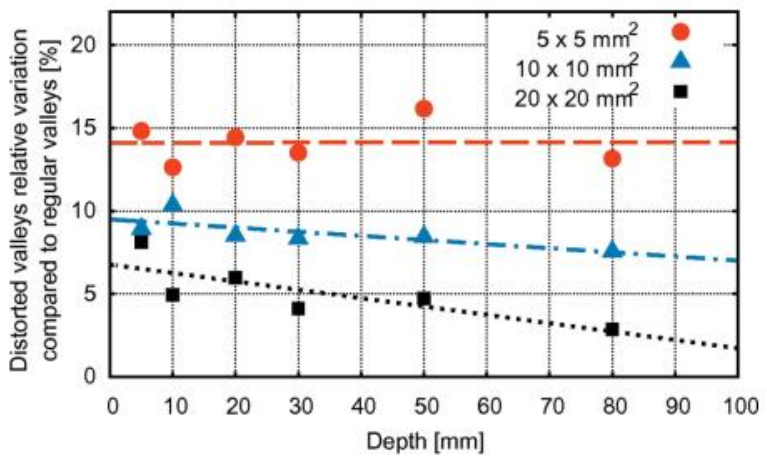

Figure 5. Relative dose variation of regular and distorted valleys for three different field sizes and at 295 different depths in the phantom. Five central valleys of each field were considered. The linear fits are guides to the eye.

Table 4. Relative dose variation of distorted valleys with respect to regular valleys for three different field sizes.

\begin{tabular}{cc}
\hline Field size & Relative dose variation [\%] \\
\hline $5 \times 5 \mathrm{~mm}^{2}$ & $12.5 \%-16.0 \%$ \\
$10 \times 10 \mathrm{~mm}^{2}$ & $7.5 \%-10.5 \%$ \\
$20 \times 20 \mathrm{~mm}^{2}$ & $3.0 \%-8.0 \%$ \\
\hline
\end{tabular}

\subsection{Radiation leakage through the multislit collimator blades}

$\mathrm{MC}$ simulations indicate that the extra peak dose contribution due to the radiation leakage through the MSC blades for the three different field sizes, the three different spectrum configurations, and at all phantom depths is, on average, equal to $0.2 \%$, with variations between $0.1 \%$ and $0.3 \%$ due to statistical fluctuations.

By contrast, valley doses are significantly affected by the radiation leakage contribution. Figure 6 presents an overview of valley depth dose profiles and related PVDRs for the three field sizes and the conventional MRT spectrum. Similar trends are obtained using the pre-clinical and clinical spectrum.

Figure 7 presents the valley dose and PVDR relative variation between simulations without and with radiation leakage. Focusing on the data at $20 \mathrm{~mm}$ depth, where reference dosimetry is performed, around $5 \%$ valley dose increase is occurring for the $20 \times 20 \mathrm{~mm}^{2}$ field, $10 \%$ for the $10 \times 10 \mathrm{~mm}^{2}$ field and $18 \%$ for the $5 \times 5 \mathrm{~mm}^{2}$ field using the conventional spectrum. Using the clinical spectrum, around $10 \%$ valley dose increase occurs for the $20 \times 20 \mathrm{~mm}^{2}$ field, $17 \%$ for the $10 \times 10 \mathrm{~mm}^{2}$ field and $30 \%$ for the $5 \times 5 \mathrm{~mm}^{2}$ field. An intermediate case is obtained when the pre-clinical MRT spectrum is used for irradiations. Even more 
significant differences are observed at the phantom surface where the relative dose increase is about twice as high as at $20 \mathrm{~mm}$ depth. PVDRs are consequently affected and an overall reduction is observed at all phantom depths. Again, PVDR reduction is stronger for small fields.
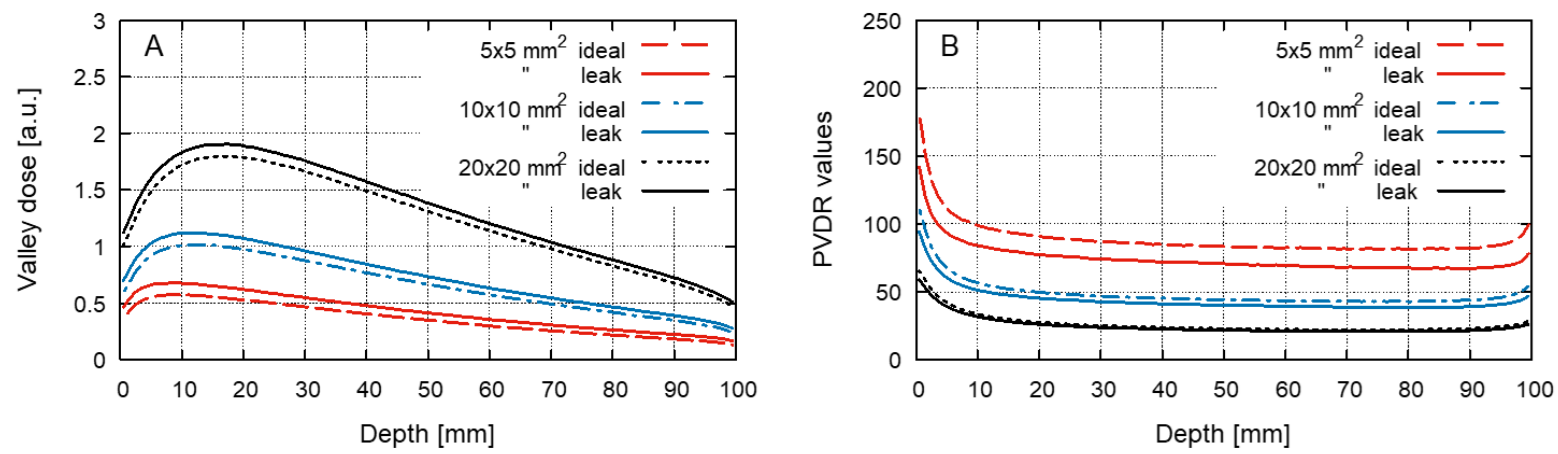

Figure 6. Influence of the MSC leakage for different field sizes and the conventional MRT spectrum. Panel $A$ and $B$ report the valley depth dose profile (left) and PVDR values (right) without and with radiation 320 leakage.
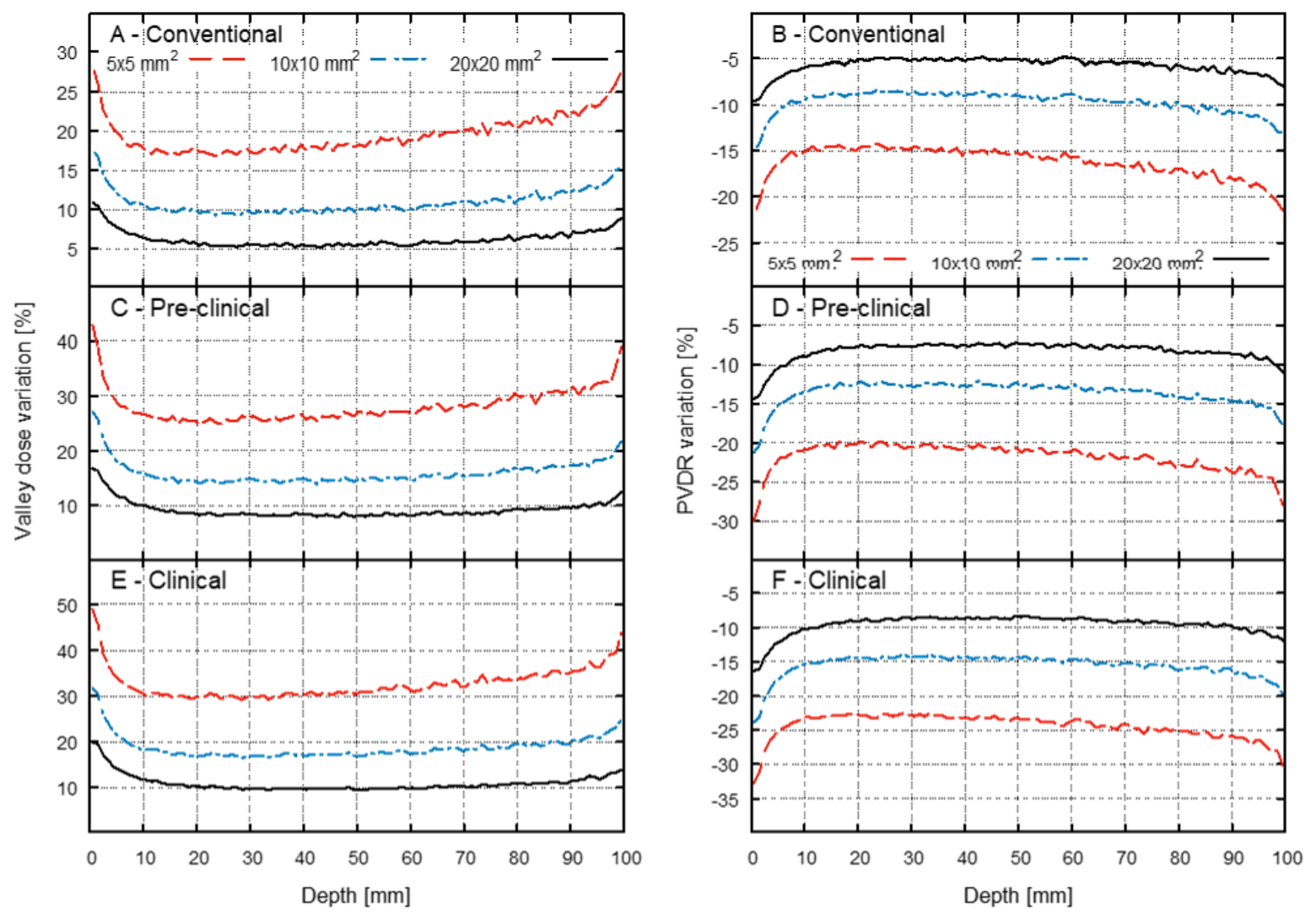
Figure 7. Influence of the MSC leakage for different field sizes, reported as relative change of the valley depth dose profile (left panels) and of PVDR values (right panels) without and with radiation leakage. Panel

$325 \quad A$ and B (top): conventional MRT spectrum; Panel C and D (center) pre-clinical MRT spectrum; Panel E and $F$ (bottom) clinical MRT spectrum.

Comparing PVDRs for a fixed field size and considering the three different spectra, the use of the clinical MRT spectrum may not be the most effective solution to increase PVDR values. In fact, for the $5 \times 5 \mathrm{~mm}^{2}$ field the PVDRs obtained from simulations using the clinical MRT spectrum and including the MSC leakage are lower than the ones obtained with the conventional MRT spectrum. The PVDR reduction is around 5\% at $20 \mathrm{~mm}$ depth and $10 \%$ at the phantom surface. When considering the $10 \times 10 \mathrm{~mm}^{2}$ field the use of harder spectra has a negligible impact on PVDR values compared to the conventional MRT spectrum at 20 $\mathrm{mm}$ depth. The advantage of using the pre-clinical or clinical MRT spectrum is visible for the $20 \times 20 \mathrm{~mm}^{2}$ field where PVDR values are $5 \%$ higher at $20 \mathrm{~mm}$ depth and $2 \%$ at the phantom surface.

335 The small fraction of photons directly impinging on the MSC but not absorbed inside the metal become primary photons for the valley region contributing with an extra dose deposition. The amount of leakage radiation is a constant fraction of the radiation impinging on the MSC absorbers, while the dose deposited into valley regions due to photon scattering inside the phantom is smaller for a small field size, as already mentioned in section 3.1. Also in this case, the additional dose contribution is more significant in a small 340 field. Moreover, the radiation scattering contribution inside the phantom close to its surface is smaller than in the center; therefore, any external dose contribution is more significant at the phantom's entrance, as visible in the relative variation of the valley dose with depth (see Figure 7).

\subsection{Analysis of alternative materials for a novel multislit collimator}

With the purpose to decrease the contribution of leakage radiation into valley regions, WC alloy and pure W were studied in dedicated Geant4 simulations as possible alternative to the WF20 alloy of the ID17 MSC. Peak and valley depth dose profiles and the corresponding PVDRs were calculated for different field sizes and MRT spectra.

As expected, peak doses are minimally affected using different MSCs. On the contrary, valley doses change significantly. Figure 8 presents the depth dose variation for a $5 \times 5 \mathrm{~mm}^{2}$ radiation field of the clinical MRT spectrum considering the three different MSCs and comparing them with the ideal case of complete absence of radiation leakage defined in section 2.3. It is clearly visible in Figure 8(A) that the use of a pure tungsten MSC provides a valley dose profile closer to the ideal case compared to the use of the WF20 alloy. 
Figure 9 presents the valley dose and PVDR relative variation between simulations without and with radiation leakage for different MSC materials, using the clinical spectrum. For a $5 \times 5 \mathrm{~mm}^{2}$ field, the valley dose at $20 \mathrm{~mm}$ depth is increased by $6 \%$ using a MSC made of $\mathrm{W}$, while it is increased by $30 \%$ using the WF20 material. The reduction by a factor of 5 of the relative dose variations is almost constant at all depths inside the phantom, and PVDRs reflect the same trend. In the case of a $20 \times 20 \mathrm{~mm}^{2}$ field, the remaining leakage contribution of radiation using a MSC made of W increases valley doses no more than $2 \%$ inside the phantom and no more than $4 \%$ close to the surface. These values are around 5 times lower compared to the use of a MSC made of WF20. Table 5 summarizes the valley dose and PVDR relative variation between simulations without and with radiation leakage considering the three field sizes, spectrum configurations and MSC materials. Values at $1 \mathrm{~mm}$ depth were considered to highlight the maximum variations occurring close to the surface of the phantom; values at $20 \mathrm{~mm}$ depth were considered because reference dosimetry in MRT is performed at this position.
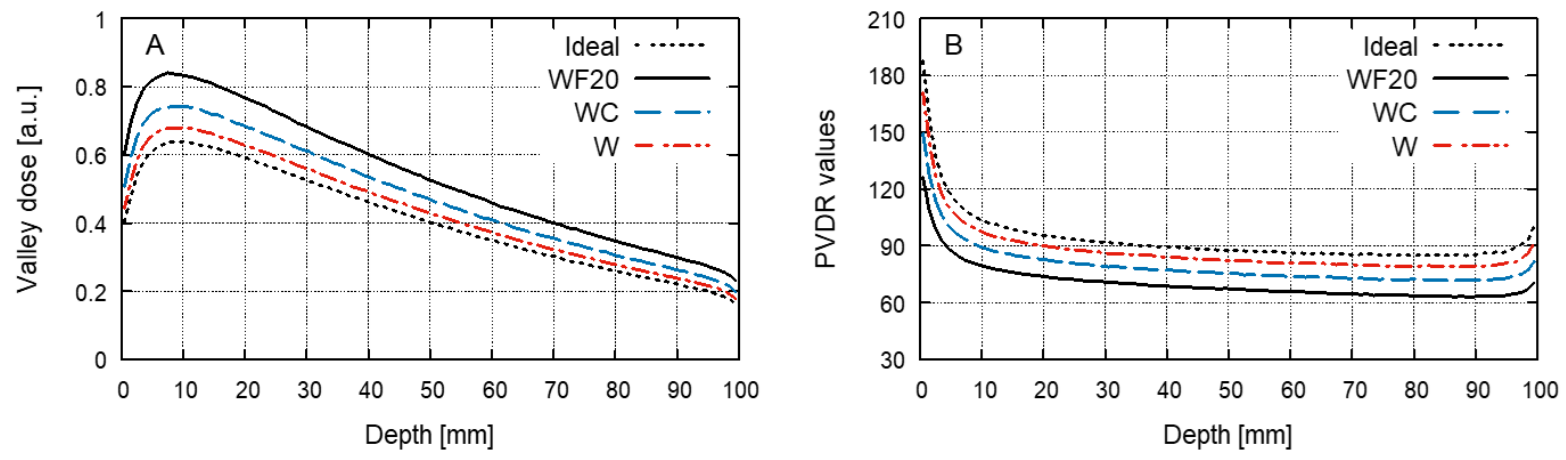

Figure 8. Influence of different MSC materials on the radiation leakage for a $5 \times 5 \mathrm{~mm}^{2}$ field, using the clinical spectrum. Panel $A$ and $B$ report the valley depth dose profile (left) and PVDR values (right) without and with radiation leakage. 

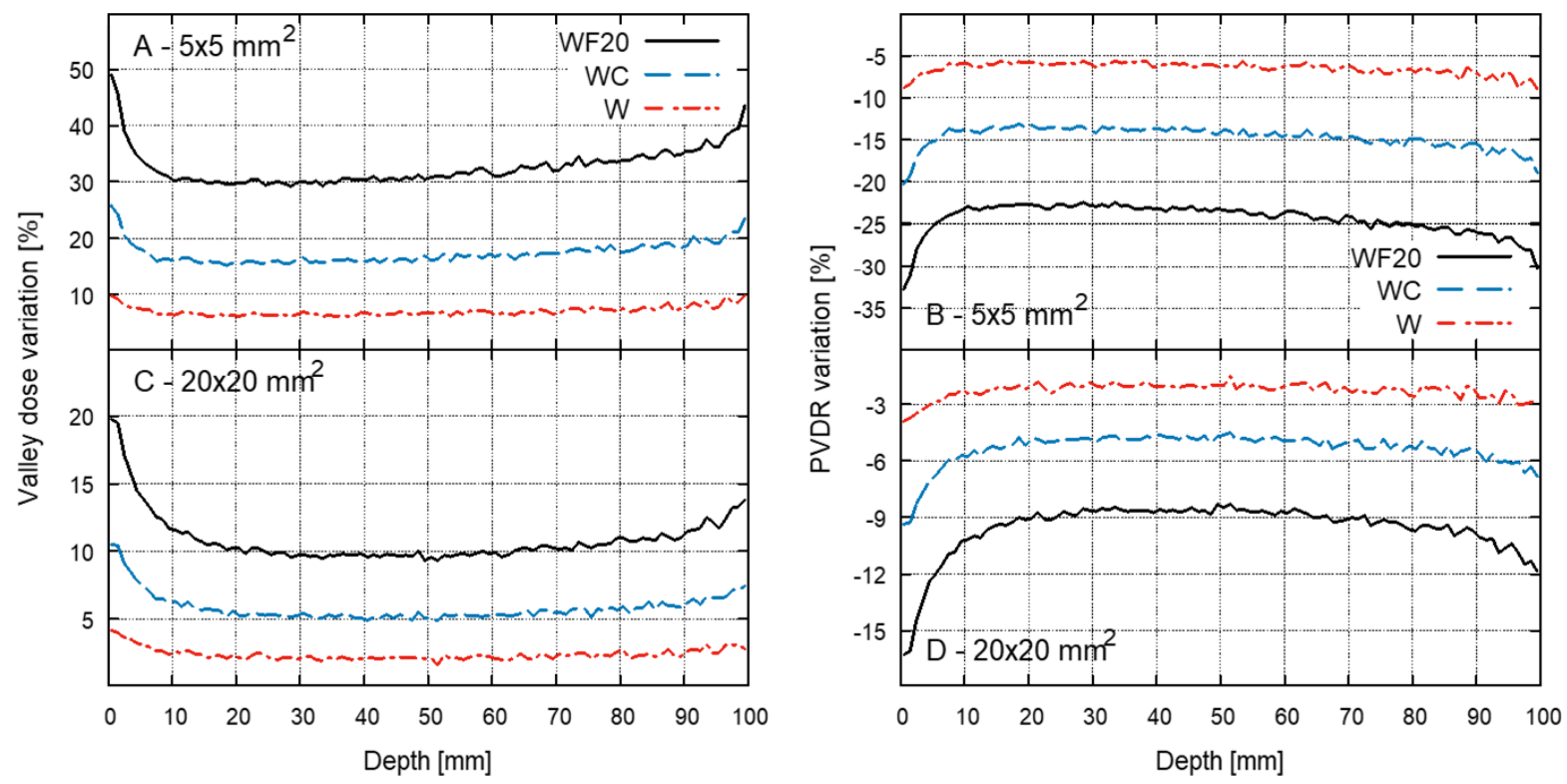

Figure 9. Influence of different MSC materials on the radiation leakage using the clinical spectrum. Left panels report the relative change (without and with radiation leakage) of the valley depth dose profile, right panels the PVDR values. Panel $A$ and $B$ (top): $5 \times 5 \mathrm{~mm}^{2}$ field; panel $C$ and D (bottom): $20 \times 20 \mathrm{~mm}^{2}$ field.

Table 5. Valley dose and PVDR relative variation between simulations without and with radiation leakage considering the three different spectrum configurations and MSC materials. Simulations without radiation leakage were chosen as reference data set. For each of the three field sizes, variations at $1 \mathrm{~mm}$ and $20 \mathrm{~mm}$ depth are reported.

\begin{tabular}{|c|c|c|c|c|c|c|c|c|c|c|c|c|}
\hline \multirow{4}{*}{$\begin{array}{c}\text { MSC } \\
\text { material }\end{array}$} & \multicolumn{12}{|c|}{ Conventional spectrum } \\
\hline & \multicolumn{6}{|c|}{ Valley dose } & \multicolumn{6}{|c|}{ PVDR } \\
\hline & \multicolumn{2}{|c|}{$5 \times 5 \mathrm{~mm}^{2}$ field } & \multicolumn{2}{|c|}{$10 \times 10 \mathrm{~mm}^{2}$ field } & \multicolumn{2}{|c|}{$20 \times 20 \mathrm{~mm}^{2}$ field } & \multicolumn{2}{|c|}{$5 \times 5 \mathrm{~mm}^{2}$ field } & \multicolumn{2}{|c|}{$10 \times 10 \mathrm{~mm}^{2}$ field } & \multicolumn{2}{|c|}{$20 \times 20 \mathrm{~mm}^{2}$ field } \\
\hline & $1 \mathrm{~mm} *$ & $20 \mathrm{~mm}^{*}$ & $1 \mathrm{~mm}$ & $20 \mathrm{~mm}$ & $1 \mathrm{~mm}$ & $20 \mathrm{~mm}$ & $1 \mathrm{~mm}$ & $20 \mathrm{~mm}$ & $1 \mathrm{~mm}$ & $20 \mathrm{~mm}$ & $1 \mathrm{~mm}$ & $20 \mathrm{~mm}$ \\
\hline WF20 & $28 \%$ & $17 \%$ & $18 \%$ & $10 \%$ & $11 \%$ & $6 \%$ & $-22 \%$ & $-15 \%$ & $-15 \%$ & $-9 \%$ & $-10 \%$ & $-5 \%$ \\
\hline WC & $14 \%$ & $9 \%$ & $9 \%$ & $5 \%$ & $6 \%$ & $3 \%$ & $-13 \%$ & $-9 \%$ & $-8 \%$ & $-5 \%$ & $-5 \%$ & $-3 \%$ \\
\hline \multirow[t]{2}{*}{ W } & $5 \%$ & $3 \%$ & $3 \%$ & $2 \%$ & $2 \%$ & $1 \%$ & $-5 \%$ & $-3 \%$ & $-3 \%$ & $-2 \%$ & $-2 \%$ & $-1 \%$ \\
\hline & \multicolumn{12}{|c|}{ Pre-clinical spectrum } \\
\hline WF20 & $43 \%$ & $25 \%$ & $27 \%$ & $14 \%$ & $17 \%$ & $8 \%$ & $-30 \%$ & $-20 \%$ & $-21 \%$ & $-12 \%$ & $-14 \%$ & $-8 \%$ \\
\hline WC & $22 \%$ & $13 \%$ & $14 \%$ & $7 \%$ & $9 \%$ & $4 \%$ & $-18 \%$ & $-12 \%$ & $-12 \%$ & $-7 \%$ & $-8 \%$ & $-4 \%$ \\
\hline \multirow[t]{2}{*}{ W } & $9 \%$ & $5 \%$ & $6 \%$ & $3 \%$ & $3 \%$ & $2 \%$ & $-8 \%$ & $-5 \%$ & $-5 \%$ & $-3 \%$ & $-3 \%$ & $-2 \%$ \\
\hline & \multicolumn{12}{|c|}{ Clinical spectrum } \\
\hline WF20 & $50 \%$ & $30 \%$ & $32 \%$ & $17 \%$ & $20 \%$ & $10 \%$ & $-33 \%$ & $-23 \%$ & $-24 \%$ & $-14 \%$ & $-16 \%$ & $-9 \%$ \\
\hline WC & $26 \%$ & $16 \%$ & $17 \%$ & $9 \%$ & $11 \%$ & $5 \%$ & $-20 \%$ & $-13 \%$ & $-14 \%$ & $-8 \%$ & $-9 \%$ & $-5 \%$ \\
\hline W & $10 \%$ & $6 \%$ & $7 \%$ & $4 \%$ & $4 \%$ & $2 \%$ & $-9 \%$ & $-6 \%$ & $-6 \%$ & $-4 \%$ & $-4 \%$ & $-2 \%$ \\
\hline
\end{tabular}

* depth 


\section{DISCUSSION}

The production of an array of beams spatially fractionated at the micrometer scale using an MSC causes the presence of different interactions between X-ray photons and the metal, and these may significantly impact the irradiation results. Ideally, a MSC should completely stop the radiation impinging on its metal blades and only transmit unperturbed photons through the apertures. Actually, photons interacting with the inner walls of the MSC may undergo scattering and also, some photons may be transmitted through the metal blades. In both cases, dose distributions inside the target are affected by these factors and they must be considered during dose calculation and experimental dosimetry.

Characterization of the surface roughness of the MSC inner walls should be the first factor to consider and 390 be included in the description of the radiation interaction with the metal surface. In addition, even if the MSC is optimally aligned before each experimental session, sub-micrometric misalignment of the metal blades during the collimator manufacture may as well influence these interactions. These phenomena require a detailed study to understand and quantify separately the different factors. Radiochromic film dosimetry is an effective approach to recognize and evaluate the total extra contribution of dose presented in "distorted" valleys. Current MC simulations are considering photon scattering from perfectly smooth and parallel inner walls of the MSC. Under these conditions, only measured "regular" valley dose must be compared to simulated dose during benchmarks.

The second factor able to significantly affect valley dose values is the radiation leakage through the MSC metal blades. The WF20 tungsten carbide alloy of the actual ID17 MSC is able to absorb efficiently photons

400 with energies below $200 \mathrm{keV}$ but the spectra used for MRT irradiations have a component above this energy that is partially transmitted, thus increasing the valley dose. When using small field sizes in the order of $5 \times 5 \mathrm{~mm}^{2}$ in combination with the clinical MRT spectrum, the valley dose is increased by $30 \%$ $50 \%$. MSC radiation leakage must therefore always be included and accurately implemented in MC simulations.

405 Radiation leakage could be reduced by using a thicker MSC. This, however, leads to a larger inner wall surface interacting with photons, thus increasing the possibility of photon scattering. Furthermore, such a MSC is more sensitive to small misalignments of the individual blades, which in turn lead to variations in the microbeam width. The use of an alternative material is the preferred choice. A pure tungsten MSC effectively absorbs photons up to $300 \mathrm{keV}$ and drastically reduces radiation leakage. This solution is currently considered for the ID17 Biomedical beamline. 
It is worth to mention that, at the moment of the preparation of this study, the phantom dimension was chosen to be similar to the dimension used by different research groups (Day et al., 2020; Dipuglia et al., 2019). Even if this geometry may not correspond to a realistic clinical scenario, the phantom is adequate to answer to the scientific question subject of this work. Steep dose variations for valley regions are more significant at the entrance and exit of the phantom, where the contribution of the scattered radiation is weaker, and these are well visible in the figures presented in the results. The equivalence between simulated and experimental volumes was well respected to obtain a correct comparison of dosimetry data, but for more clinically relevant studies a cubic phantom of $20 \mathrm{~cm}$ width and length should be the preferable solution.

420 The datasets presented in this work are valid for some specific MRT configurations but provide valuable general guidelines for any MRT setup and the associated dosimetry protocols. Experimental valley doses at ID17 were typically higher than MC calculated values and most of the discrepancy is explained by the factors quantified in this work. A first comparison between previously irradiated film and the updated MC simulation confirms the improvements of the MC model for the pre-clinical scenario and the use of a 20 $425 \times 20 \mathrm{~mm}^{2}$ field. Table 6 presents values such as peak and valley output factor with respect to reference conditions as described in section 2.2. In the same table, PVDR values are as well reported. The agreement is in average better than $5 \%$, close to the agreement required for clinical applications that is typically in the order of $3 \%$. This is an encouraging starting point and a more exhaustive and complete benchmarking of experimental dosimetry against simulated dose is already scheduled.

430 Table 6. Output factor (OF) and PVDR values for MC simulation and radiochromic film dosimetry obtained using the pre-clinical scenario and a $20 \times 20 \mathrm{~mm}^{2}$ field.

\begin{tabular}{c|ccc|ccc|ccc}
\hline $\begin{array}{c}\text { Depth } \\
\text { [mm] }\end{array}$ & \multicolumn{3}{|c|}{ Peak dose OF } & \multicolumn{3}{c|}{ Valley dose OF } & \multicolumn{3}{c}{ PVDR values } \\
\hline 20 & MC & Film & $\begin{array}{c}\text { Relative va- } \\
\text { riation [\%] }\end{array}$ & MC & Film & $\begin{array}{c}\text { Relative va- } \\
\text { riation [\%] }\end{array}$ & MC & Film & $\begin{array}{c}\text { Relative va- } \\
\text { riation [\%] }\end{array}$ \\
\hline 40 & 0.722 & 0.711 & -1.6 & 0.0260 & 0.0266 & 2.4 & 27.8 & 26.7 & -3.8 \\
60 & 0.528 & 0.500 & -5.3 & 0.0220 & 0.0217 & -1.7 & 23.9 & 23.1 & -3.6 \\
80 & 0.281 & 0.268 & -4.8 & 0.0128 & 0.0127 & -0.8 & 22.0 & 21.1 & -4.0 \\
\hline
\end{tabular}




\section{CONCLUSIONS}

The photon interaction with a MSC for the generation of microbeams is a key point for the reliable

435 definition of applications in radiotherapy. An ideal model where all photons impinging on the metal blades of an MSC are absorbed and do not reach the target is a too strong approximation and must not be used in simulated dose calculations.

Considering the most recent studies in MRT performed at ID17, a detailed analysis of the factors influencing dose distribution in the target related to photon-MSC interactions was performed. The

440 contribution of radiation interaction with the inner walls of the MSC and radiation leakage through the absorber was characterized for three different field sizes and three different X-ray spectra. Small fields of $5 \times 5 \mathrm{~mm}^{2}$ are strongly affected by both factors because the weak scattered dose between microbeams is comparable with the parasitic dose contribution.

The use of harder and less intense spectra as defined for clinical trials leads to significant radiation leakage 445 as well for $20 \times 20 \mathrm{~mm}^{2}$ fields. The WF20 tungsten carbide blades of the ID17 MSC do not sufficiently absorb photons with energies above $200 \mathrm{keV}$ and other materials should be considered.

The quantification of the dose contributions due to the X-ray radiation interaction with the MSC is fundamental and it supports the definition of more reliable dosimetry protocols for MRT, facilitating a precise benchmarking of experimental dose values against simulated dose values.

\section{Acknowledgments}

Paolo Pellicioli acknowledges PhD scholarship funding from Université Grenoble Alpes (UGA), and Swansea University. Paolo Pellicioli further thanks the European Synchrotron Radiation Facility (ESRF), the Centre for Medical Radiation Physics, University of Wollongong (UOW), and the Initiative d'excellence (IDEX) project of the UGA for funding and support. 
Agostinelli, S., Allison, J., Amako, K., Apostolakis, J., Araujo, H., Arce, P., Asai, M., Axen, D., Banerjee, S., Barrand, G., Behner, F., Bellagamba, L., Boudreau, J., Broglia, L., Brunengo, A., Burkhardt, H., Chauvie, S., Chuma, J., Chytracek, R., Cooperman, G., Cosmo, G., Degtyarenko, P., Dell'Acqua, A., Depaola, G., Dietrich, D., Enami, R., Feliciello, A., Ferguson, C., Fesefeldt, H., Folger, G., Foppiano, F., Forti, A., Garelli, S., Giani, S., Giannitrapani, R., Gibin, D., Gomez Cadenas, J. J., Gonzalez, I., Gracia Abril, G., Greeniaus, G., Greiner, W., Grichine, V., Grossheim, A., Guatelli, S., Gumplinger, P., Hamatsu, R., Hashimoto, K., Hasui, H., Heikkinen, A., Howard, A., Ivanchenko, V., Johnson, A., Jones, F. W., Kallenbach, J., Kanaya, N., Kawabata, M., Kawabata, Y., Kawaguti, M., Kelner, S., Kent, P., Kimura, A., Kodama, T., Kokoulin, R., Kossov, M., Kurashige, H., Lamanna, E., Lampen, T., Lara, V., Lefebure, V., Lei, F., Liendl, M., Lockman, W., Longo, F., Magni, S., Maire, M., Medernach, E., Minamimoto, K., Mora de Freitas, P., Morita, Y., Murakami, K., Nagamatu, M., Nartallo, R., Nieminen, P., Nishimura, T., Ohtsubo, K., Okamura, M., O'Neale, S., Oohata, Y., Paech, K., Perl, J., Pfeiffer, A., Pia, M. G., Ranjard, F., Rybin, A., Sadilov, S., di Salvo, E., Santin, G., Sasaki, T., Savvas, N., Sawada, Y., Scherer, S., Sei, S., Sirotenko, V., Smith, D., Starkov, N., Stoecker, H., Sulkimo, J., Takahata, M., Tanaka, S., Tcherniaev, E., Safai Tehrani, E., Tropeano, M., Truscott, P., Uno, H., Urban, L., Urban, P., Verderi, M., Walkden, A., Wander, W., Weber, H., Wellisch, J. P., Wenaus, T., Williams, D. C., Wright, D., Yamada, T., Yoshida, H. \& Zschiesche, D. (2003). Nucl. Instruments Methods Phys. Res. Sect. A Accel. Spectrometers, Detect. Assoc. Equip. 506, 250-303.

Anschel, D. J., Bravin, A. \& Romanelli, P. (2011). Neurosurg. Rev. 34, 133-142.

Archer, D. W. (1998). Collimator for producing an array of microbeams, U.S. Patent No. 5771270.

Bartzsch, S., Corde, S., Crosbie, J. C., Day, L., Donzelli, M., Krisch, M., Lerch, M., Pellicioli, P., Smyth, L. M. L. \& Tehei, M. (2020). Phys. Med. Biol. 65,.

Bartzsch, S., Cummings, C., Eismann, S. \& Oelfke, U. (2016). Med. Phys. 43, 6301-6308.

Bartzsch, S., Lerch, M., Petasecca, M., Oelfke, U., Bräuer-Krisch, E., Oelfke, U., Bräuer-Krisch, E., Oelfke, U., Bräuer-Krisch, E., Oelfke, U. \& Bräuer-Krisch, E. (2014). Med. Phys. 41,.

Bouchet, A., Lemasson, B., Christen, T., Potez, M., Rome, C., Coquery, N., Le Clec'H, C., Moisan, A., BräuerKrisch, E., Leduc, G., Rémy, C., Laissue, J. A., Barbier, E. L., Brun, E. \& Serduc, R. (2013). Radiother. Oncol. 108, 143-148.

Bouchet, A., Lemasson, B., Le Duc, G., Maisin, C., Bräuer-Krisch, E., Siegbahn, E. A., Renaud, L., Khalil, E., Rémy, C., Poillot, C., Bravin, A., Laissue, J. A., Barbier, E. L. \& Serduc, R. (2010). Int. J. Radiat. Oncol. Biol. Phys. 78, 1503-1512.

Bräuer-Krisch, E., Bravin, A., Lerch, M., Rosenfeld, A., Stepanek, J., Di Michiel, M. \& Laissue, J. A. (2003). Med Phys. 30, 583-589.

Bräuer-Krisch, E., Bravin, A., Zhang, L., Siegbahn, E., Stepanek, J., Blattmann, H., Slatkin, D. N., Gebbers, J. O., Jasmin, M. \& Laissue, J. A. (2005). Rev. Sci. Instrum. 76,.

Bräuer-Krisch, E., Requardt, H., Brochard, T., Berruyer, G., Renier, M., Laissue, J. A. \& Bravin, A. (2009). Rev. Sci. Instrum. 80,.

Bräuer-Krisch, E., Serduc, R., Siegbahn, E. A., Le Duc, G., Prezado, Y., Bravin, A., Blattmann, H. \& Laissue, J. A. (2010). Mutat. Res. - Rev. Mutat. Res. 704, 160-166. 
Cornelius, I., Guatelli, S., Fournier, P., Crosbie, J. C., Sanchez Del Rio, M., Bräuer-Krisch, E., Rosenfeld, A. \& Lerch, M. (2014). J. Synchrotron Radiat. 21, 518-528.

Crosbie, J. C., Anderson, R. L., Rothkamm, K., Restall, C. M., Cann, L., Ruwanpura, S., Meachem, S., Yagi, N., Svalbe, I., Lewis, R. A., Williams, B. R. G. \& Rogers, P. A. W. (2010). Int. J. Radiat. Oncol. Biol. Phys. 77, 886-894.

Crosbie, J. C., Fournier, P., Bartzsch, S., Donzelli, M., Cornelius, I., Stevenson, A. W., Requardt, H. \& BräuerKrisch, E. (2015). J. Synchrotron Radiat. 22, 1035-1041.

Day, L. R. J., Pellicioli, P., Gagliardi, F., Barnes, M., Smyth, L. M. L., Butler, D., Livingstone, J., Stevenson, A. W., Lye, J., Poole, C. M., Hausermann, D., Rogers, P. A. W. \& Crosbie, J. C. (2020). Phys. Medica. 77, 64-74.

505 Dilmanian, F. A., Button, T. M., le Duc, G., Zhong, N., Peña, L. A., Smith, J. A. L., Martinez, S. R., Bacarian, T., Tammam, J., Ren, B., Farmer, P. M., Kalef-Ezra, J., Micca, P. L., Nawrocky, M. M., Niederer, J. A., Recksiek, F. P., Fuchs, A. \& Rosen, E. M. (2004). Neuro. Oncol. 4, 26-38.

Dipuglia, A., Cameron, M., Davis, J. A., Cornelius, I. M., Stevenson, A. W., Rosenfeld, A. B., Petasecca, M., Corde, S., Guatelli, S. \& Lerch, M. L. F. F. (2019). Sci. Rep. 9, 1-14.

Donzelli, M., Brauer-Krisch, E., Oelfke, U., Wilkens, J. J. \& Bartzsch, S. (2018). Phys. Med. Biol. 63,.

Fernandez-Palomo, C., Trappetti, V., Potez, M., Pellicioli, P., Krisch, M., Laissue, J. \& Djonov, V. (2020). Cancers (Basel). 12, 1-13.

Fournier, P., Cornelius, I., Donzelli, M., Requardt, H., Nemoz, C., Petasecca, M., Bräuer-Krisch, E., Rosenfeld, A. \& Lerch, M. (2016). J. Synchrotron Radiat. 23, 1180-1190.

Fournier, P., Crosbie, J. C., Cornelius, I., Berkvens, P., Donzelli, M., Clavel, A. H., Rosenfeld, A. B., Petasecca, M., Lerch, M. L. F. \& Bräuer-Krisch, E. (2016). Phys. Med. Biol. 61, N349-N361.

Griffin, R. J., Koonce, N. A., Dings, R. P. M., Siegel, E., Moros, E. G., Bruer-Krisch, E. \& Corry, P. M. (2012). Radiat. Res. 177, 804-812.

Hadsell, M., Cao, G., Zhang, J., Burk, L., Schreiber, T., Schreiber, E., Chang, S., Lu, J. \& Zhou, O. (2014). Med. Phys. 41,.

Hubbell, J. H. \& Seltzer, S. M. (1996). NIST: X-Ray Mass Attenuation Coefficients.

Ibahim, M. J., Crosbie, J. C., Yang, Y., Zaitseva, M., Stevenson, A. W., Rogers, P. A. W. \& Paiva, P. (2014). PLoS One. 9,.

Ibahim, M. J., Yang, Y., Crosbie, J. C., Stevenson, A., Cann, L., Paiva, P. \& Rogers, P. A. (2016). Radiat. Res. 185, 60-68.

Laissue, J. A., Bartzsch, S., Blattmann, H., Bräuer-Krisch, E., Bravin, A., Dalléry, D., Djonov, V., Hanson, A. L., Hopewell, J. W., Kaser-Hotz, B., Keyriläinen, J., Laissue, P. P., Miura, M., Serduc, R., Siegbahn, A. E. \& Slatkin, D. N. (2013). Radiother. Oncol. 106, 106-111.

Laissue, J. A., Blattmann, H. \& Slatkin, D. N. (2012). Z. Med. Phys. 22, 90-99.

Laissue, J. A., Geiser, G., Spanne, P. O., Dilmanian, F. A., Gebbers, J.-O. O., Geiser, M., Wu, X.-Y. Y., Makar, M. S., Micca, P. L., Nawrocky, M. M., Joel, D. D. \& Slatkin, D. N. (1998). Int. J. Cancer. 78, 654-660. 
Martínez-Rovira, I., Sempau, J. \& Prezado, Y. (2012). Med. Phys. 39, 119-131.

Musolino, S. V. \& IAEA TRS-398 (2001). Absorbed Dose Determination in External Beam Radiotherapy: An International Code of Practice for Dosimetry Based on Standards of Absorbed Dose to Water; Technical Reports Series No. 398,.

Nettelbeck, H., Takacs, G. J., Lerch, M. L. F. F. \& Rosenfeld, A. B. (2009). Med. Phys. 36, 447-456.

Ocadiz, A., Livingstone, J., Donzelli, M., Bartzsch, S., Nemoz, C., Kefs, S., Pellicioli, P., Giraud, J. Y., Balosso, J., Krisch, M., Bräuer-krisch, E., Serduc, R. \& Adam, J. F. (2019). Phys. Medica. 65, 227-237.

Pellicioli, P., Bartzsch, S., Donzelli, M., Krisch, M. \& Bräuer-Krisch, E. (2019). Phys. Medica. 65, 106-113.

Pouyatos, B., Nemoz, C., Chabrol, T., Potez, M., Bräuer, E., Renaud, L., Pernet-Gallay, K., Estève, F., David, O., Kahane, P., Laissue, J. A., Depaulis, A. \& Serduc, R. (2016). Sci. Rep. 6,.

Pouyatos, B., Serduc, R., Chipaux, M., Chabrol, T., Bräuer-Krisch, E., Nemoz, C., Mathieu, H., David, O., Renaud, L., Prezado, Y., Laissue, J. A., Estève, F., Charpier, S. \& Depaulis, A. (2013). Neurobiol. Dis. 51, 152-160.

Requardt, H., Renier, M., Brochard, T., Bräuer-Krisch, E., Bravin, A. \& Suortti, P. (2013). J. Phys. Conf. Ser. 425,.

Del Río, M. S. \& Dejus, R. J. (2004). AIP Conf. Proc. 705, 784-787.

Romanelli, P. \& Bravin, A. (2011). Neurol. Res. 33, 825-831.

Salvat, F., Fernández-Vera, J. \& Sempau, J. (2009). Simulation, Vol. p. 324.

550 Van Der Sanden, B., Bräuer-Krisch, E., Siegbahn, E. A., Ricard, C., Vial, J. C. \& Laissue, J. (2010). Int. J. Radiat. Oncol. Biol. Phys. 77, 1545-1552.

Schültke, E., Juurlink, B. H. J., Ataelmannan, K., Laissue, J., Blattmann, H., Bräuer-Krisch, E., Bravin, A., Minczewska, J., Crosbie, J., Taherian, H., Frangou, E., Wysokinsky, T., Chapman, L. D., Griebel, R. \& Fourney, D. (2008). Eur. J. Radiol. 68, S142-S146.

Serduc, R., Bouchet, A., Bräuer-Krisch, E., Laissue, J. A., Spiga, J., Sarun, S., Bravin, A., Fonta, C., Renaud, L., Boutonnat, J., Siegbahn, E. A., Estève, F. \& Le Duc, G. (2009). Phys. Med. Biol. 54, 6711-6724.

Slatkin, D. N., Blattmann, H., Wagner, H. P., Glotzer, M. A., Laissue, J. A., Grotzer, M. A., Laissue, J. A., Blattmann, H., Wagner, H. P., Grotzer, M. A., Slatkin, D. N., Blattmann, H., Wagner, H. P., Glotzer, M. A. \& Laissue, J. A. (2007). Dev. Med. Child Neurol. 49, 577-581.

Slatkin, D. N., Dilmanian, F. A., Nawrocky, M. M., Spanne, P., Gebbers, J. O., Archer, D. W. \& Laissue, J. A. (1995). Rev. Sci. Instrum. 66, 1459-1460.

Slatkin, D. N., Spanne, P., Dilmanian, F. A., Gebberst, J.-O., Laissue, J. A., Gebbers, J. O., Laissue, J. A., Gebberst, J.-O., Laissue, J. A., Gebbers, J. O. \& Laissue, J. A. (1995). Proc. Natl. Acad. Sci. U. S. A. 92, 8783-8787.

565 Slatkin, D. N., Spanne, P., Dilmanian, F. A., Sandbora, M., Slatkin, Slatkin, D. N., Spanne, P., Dilmanian, F. A. \& Sandbora, M. (1992). Medica Phys. 19, 1395-1400.

Smyth, L. M. L., Donoghue, J. F., Ventura, J. A., Livingstone, J., Bailey, T., Day, L. R. J., Crosbie, J. C. \& Rogers, P. A. W. (2018). Sci. Rep. 8, 1-11. 
Studer, F., Serduc, R., Pouyatos, B., Chabrol, T., Bräuer-Krisch, E., Donzelli, M., Nemoz, C., Laissue, J. A., Estève, F. \& Depaulis, A. (2015). Phys. Medica. 31, 607-614.

Zeman, W., Curtis, H. J. \& Baker, C. P. (1961). Radiat. Res. 15, 496.

Zippo, A. G., Bertoli, G., Riccardi, M. P., Valente, M., Bräuer-Krisch, E., Caramenti, G. C., Requardt, H., del Grosso, V., Coan, P., Bravin, A. \& Biella, G. E. M. (2019). BioRxiv.

(1957). Science (80-. ). 125, 18-22.

575 